\title{
AUFSATZ
}

\section{Parlamentswahl in der Diktatur: Eine Analyse des Wählerverhaltens auf Ebene der Land- und Stadtkreise bei der Reichstagswahl vom 12. November 1933}

\author{
Marc Debus
}

Der politische Prozess in der Weimarer Republik ist aus vielfältiger Perspektive beleuchtet worden, etwa im Hinblick auf die Eigenschaften institutioneller Faktoren wie der Kompetenzen des Reichspräsidenten oder die Auswirkungen des reinen Verhältniswahlsystems auf die Struktur des Parteiensystems, die Stabilität der Regierungen und die Inhalte von Politik. ${ }^{1}$ Die Politische Soziologie hat sich intensiv mit den möglichen Determinanten des individuellen Wahlverhaltens der Bürger bei den Wahlen zu Nationalversammlung und Reichstag in der Zeit von Januar 1919 bis März 1933 beschäftigt und hierbei insbesondere die Gründe für den elektoralen Aufstieg der Nationalsozialisten (NSDAP) oder die Gründe für den Anstieg ihrer Mitgliederzahl näher untersucht. ${ }^{2}$ Während frühere Studien die Ursachen für den nahezu kontinuierlich anwachsenden Stimmenanteil der NSDAP in der steigenden Arbeitslosigkeit - und auch den Arbeitslosen selbst - sowie den mit der wirtschaftlichen Krise verbundenen Ängsten in der bürgerlichen Mittelschicht gesehen haben ${ }^{3}$, konnte Jürgen W. Falter ${ }^{4}$ in seiner bahnbrechenden Studie zeigen, dass es vor allem die protestantisch geprägten Mittelschichtangehörigen waren, die die NSDAP wählten, hingegen signifikant weniger die Arbeitslosen oder Industriearbeiter. Katholiken erwiesen sich Falters Befunden zufolge - als nahezu „immun“ gegenüber einer Wahl der Nationalsozialis-

1 Vgl. Dirk Berg-Schlosser, Das Scheitern der Weimarer Republik - Bedingungen der Demokratie im europäischen Vergleich, in: Historical Social Research, 20. Jg. (1995), H. 1, S. 3 - 30; Eberhard Kolb, Die Weimarer Republik, München 2002; Peter Longerich, Deutschland 1918-1933. Die Weimarer Republik, Hannover 1995; Hans Mommsen, Aufstieg und Untergang der Republik von Weimar, München 2004; Hagen Schulze, Weimar: Deutschland 1917-1933, Berlin 1998; Heinrich August Winkler, Weimar 1918-1933: Die Geschichte der ersten deutschen Demokratie, München 2005; Eckhard Jesse, Das Parteiensystem des Kaiserreichs und der Weimarer Republik, in: Oskar Niedermayer (Hrsg.), Handbuch Parteienforschung, Wiesbaden 2013, S. 685 - 710, S. $695-702$.

2 Vgl. etwa Seymour Martin Lipset, „Fascism“ - Left, Right, and Center, in: ders., Political Man: The Social Bases of Politics, Baltimore 1981, S. 127 - 152; Walter Dean Burnham, Political Immunization and Political Confessionalism: The United States and Weimar Germany, in: The Journal of Interdisciplinary History, 3. Jg. (1972), H. 1, S. 1 - 30; Brian Ault / William Brustein, Joining the Nazi Party Explaining the Political Geography of NSDAP Membership, 1925-1933, in: American Behavioral Scientist, 41. Jg. (1998), H. 9, S. 1304 - 1323; Jürgen W. Falter, Hitlers Wähler, München 1991; Ernest Hamburger / Peter Pulzer, Jews as Voters in the Weimar Republic, in: The Leo Baeck Institute Yearbook, 30. Jg. (1985), H. 1, S. 3 - 66; John O'Loughlin / Colin Flint / Luc Anselin, The Geography of the Nazi Vote: Context, Confession, and Class in the Reichstag Election of 1930, in: Annals of the Association of American Geographers, 84. Jg. (1994), H. 3, S. $351-380$.

3 Vgl. Seymour Martin Lipset, a.a.O. (Fn. 2).

4 Vgl. Jürgen W. Falter, a.a.O. (Fn. 2). 
ten. ${ }^{5}$ Die Studie von Falter wie auch andere Untersuchungen zu den Wahlen zur Nationalversammlung und zum Reichstag von 1919 bis 1933 beziehen jedoch die Reichstagswahl vom 12. November 1933 nicht mit ein, die gleichzeitig mit der Volksabstimmung über den Austritt Deutschlands aus dem Völkerbund stattfand. Dies hat einen sehr guten Grund: Die Wahl fand knapp zehn Monate nach dem Amtsantritt Hitlers als Reichskanzler statt, die Transformation zur Parteidiktatur war bereits vollzogen, und es bestand dementsprechend nicht die Möglichkeit, zwischen verschiedenen Listen mit Kandidaten auszuwählen. Vielmehr gab es eine Einheitsliste der NSDAP, auf der auch wenige ehemalige Mitglieder der zu diesem Zeitpunkt bereits selbst aufgelösten nationalliberalen Deutschen Volkspartei (DVP), nationalkonservativen Deutschnationalen Volkspartei (DNVP) und des katholischen Zentrums platziert waren. Eine solche Konstellation lässt die Analyse von Wahlergebnissen und Wählerverhalten als nicht besonders fruchtbar erscheinen. Hingegen ergibt sich aus einer deskriptiven Betrachtung der Verteilung von Wahlbeteiligung und der Zustimmung zur NSDAP-Einheitsliste - bei einer Wahlbeteiligung von 95,2 Prozent der Wahlberechtigten stimmten 92,1 Prozent für die nationalsozialistische Kandidatenliste, während 7,9 Prozent ihren Stimmzettel ungültig machten, das heißt nicht mit ,ja“ stimmten $^{6}$ - deutliche Varianz über die Städte und Landkreise des Deutschen Reiches. ${ }^{7}$ So lag beispielsweise in Lübeck der Anteil der ungültigen Stimmen an allen abgegebenen Stimmen bei 21,8 Prozent, in Hamburg bei 16,8 Prozent und in Leipzig bei 15,1 Prozent. $^{8}$ Auch im katholisch geprägten Aachen stimmten 14,4 Prozent der Wähler nicht für die NSDAP-Liste und machten damit ihren Stimmzettel ungültig, was deutlich über dem landesweiten Durchschnittswert von knapp acht Prozent der Wähler lag, die der Einheitsliste keine Zustimmung erteilten. Hingegen fiel der Wert im Landkreis Kassel oder in der Stadt Kaiserslautern mit 0,7 beziehungsweise 0,1 Prozent deutlich niedriger aus. Summiert man den Anteil der Nichtwähler und der abgegebenen ungültigen Stimmen an allen Wahlberechtigten auf, ergeben sich Anteilswerte von 29,1 beziehungsweise 22,2 Prozent der Stimmberechtigten in den Hansestädten Lübeck und Hamburg sowie 20,2 Prozent in Leipzig und 19,4 Prozent in Aachen, die der NSDAP-Einheitsliste aufgrund von Nichtwahl oder einer Ungültigmachung des Stimmzettels die Zustimmung direkt oder indirekt verweigerten. Im Landkreis Bomst, der zur preußischen Provinz Posen gehörte, oder in den nordhessischen Landkreisen Ziegenhain und Hofgeismar lagen die Werte mit 0,5 beziehungsweise mit jeweils 0,9 Prozent hingegen wesentlich niedriger als im Landesdurchschnitt von 12,2 Prozent - nahezu alle Wahlberechtigten gingen dort zur Wahl und stimmten für die Liste der NSDAP.

Ziel dieses Beitrags ist die Beantwortung der Frage, ob und inwiefern die (sozial-)strukturellen Merkmale und damit Indikatoren für die Milieustruktur der Städte und Landkreise

5 Vgl. ebenda, S. 188.

6 Die Option, die Liste - etwa mit einem Kreuz bei einer Wahlmöglichkeit „nein“ - explizit abzulehnen, war nicht gegeben. Der Stimmzettel sah lediglich die Möglichkeit vor, die NSDAP-Liste anzukreuzen oder nicht.

7 Vgl. Hans-Ulrich Wehler, Der Nationalsozialismus. Bewegung, Führerschaft, Verbrechen, München 2009, S. 72.

8 Diese und die folgenden Werte sind eigene Berechnungen auf der Grundlage der Datenzusammenstellung des Statistischen Reichsamts. Siehe Statistisches Reichsamt, Die Wahlen zum Reichstag und die Volksabstimmung am 12. November 1933 sowie die Volksabstimmung am 19. August 1934, Berlin 1935. 
des Deutschen Reiches helfen können, die unterschiedliche Verteilung von Wahlbeteiligung und ungültigen Stimmen bei dieser „Wahl“ auf regionaler Ebene zu erklären, die zwar bereits während der NS-Diktatur stattfand, bei der allerdings die Nationalsozialisten noch keine systematische Wahlbeeinflussung vorgenommen hatten beziehungsweise vornehmen konnten. ${ }^{9}$ Die oben aufgeführten, beispielhaften Ergebnisse deuten zwar darauf hin, dass in industriell geprägten Gebieten und damit in Hochburgen von Sozialdemokraten und Kommunisten wie auch in katholischen Gegenden die direkt wie indirekt bei der Reichstagswahl im November 1933 geäußerte Opposition gegenüber der NSDAP-Einheitsliste deutlich stärker ausgeprägt war als in protestantisch und ländlich geprägten Regionen. Eine systematische Untersuchung dieser Zusammenhänge steht jedoch noch aus. ${ }^{10}$

\section{Soziale Milieus und Wählerverhalten bei Reichstagswablen in der Weimarer Republik}

Aufgrund fehlender Umfragedaten oder Wahltagsbefragungen („exit polls“) ist die Analyse der Bestimmungsfaktoren des individuellen Wahl- und Abstimmungsverhaltens bei zeitlich weit zurückliegenden Wahlen naturgemäß schwierig. Dennoch gibt es eine Reihe von Untersuchungen zu den Mustern des Wählerverhaltens während der Weimarer Republik, die auf aggregierten Daten beruhen. ${ }^{11}$ Zentral ist dabei nach wie vor die Studie von Falter: Mit Hilfe von Wahlergebnissen auf regionaler und lokaler Ebene - den Stadt- und Landkreisen und den Gemeinden - sowie der sozialstrukturellen Eigenschaften der jeweiligen Gebietskörperschaften untersuchte er Zusammenhänge zwischen den dortigen Stimmenanteilen der Parteien und den sozio-ökonomischen Charakteristika (Anteil der Landarbeiter, der Industriearbeiter, der Selbstständigen, der Protestanten und der Katholiken) sowie den Stimmenanteilen der Parteien bei früheren Wahlen mit multivariaten Regressionsmodellen. ${ }^{12}$ Der Fokus auf die lokalen beziehungsweise regionalen Einheiten ermöglichte eine deutliche Vergrößerung der Fallzahl, so dass statistisch robuste Ergebnisse und damit Schlussfolgerungen im Hinblick auf den Einfluss der Berufsgruppenzugehörigkeit und der konfessionellen Ausrichtung der Wählerschaft gewonnen werden konnten, während gleichzeitig die Gefahr des ökologischen Fehlschlusses gemindert wurde. Falter konnte mit seiner die Reichstagswahlen von 1920 bis März 1933 umfassenden Untersuchung zeigen, dass die Stimmenanteile für die NSDAP desto niedriger ausfielen, je größer der Anteil der Bevölke-

9 Vgl. Hans-Ulrich Wehler, a.a.O. (Fn. 7), S. 72. Allerdings wurden viele Wahllokale von Truppen der Sturmabteilung (SA) bewacht und Bürger, die bislang nicht im Wahllokal erschienen waren, an manchen Orten von der SA zu Hause aufgesucht, um sie zur Stimmabgabe aufzufordern beziehungsweise direkt zum Wahllokal zu fahren. Vgl. Nico Voigtländer / Hans-Joachim Voth, Highway to Hitler, National Bureau of Economic Research Working Paper Series, Working Paper 20150, http://www.nber.org/papers/w20150 (Abruf am 16. August 2016), S. $11 \mathrm{f}$.

10 Vgl. jedoch Nico Voigtlaender / Hans-Joachim Voth, a.a.O. (Fn. 9), die eindrucksvoll anhand eines Vergleichs der Ergebnisse der Reichstagswahl vom 12. November 1933 und des Plebiszits über das Staatsoberhaupt des Deutschen Reichs am 19. August 1934 zeigen, dass die Zustimmung zum nationalsozialistischen Regime in jenen Regionen stärker anwuchs, in denen der vom NSRegime forcierte, allerdings schon in der Zeit der Weimarer Republik geplante Autobahnbau stattfand.

11 Vgl. Jürgen W. Falter, a.a.O. (Fn. 2); siehe auch Karl Rohe, Wahlen und Wählertraditionen in Deutschland, Frankfurt am Main 1992, S. 121 - 163.

12 Vgl. Jürgen W. Falter, a.a.O. (Fn. 2). 
rung war, welcher der römisch-katholischen Kirche angehörte. Auch wiesen Städte und Gemeinden, die durch einen hohen Industriearbeiteranteil charakterisiert waren, einen tendenziell niedrigeren Stimmenanteil für die Nationalsozialisten auf. Zwar gab es durchaus Industriearbeiter und Katholiken in der Wählerschaft der NSDAP, jedoch waren sie im Vergleich zu ihrem Anteil an der Gesamtbevölkerung deutlich unterrepräsentiert. ${ }^{13}$ Hingegen zeigt sich auf der Grundlage der von Falter vorgenommenen ökologischen Regressionsanalysen Evidenz dafür, dass Angehörige des so genannten „alten Mittelstandes“ - Selbstständige und mithelfende Familienangehörige - überdurchschnittlich häufig die NSDAP gewählt haben. Zudem hatte der Grad der Urbanisierung einen negativen Effekt auf das Abschneiden der NSDAP: Je höher die Einwohnerzahl einer Gebietskörperschaft war, desto geringer fiel der Stimmenanteil für die Nationalsozialisten - insbesondere in industriell geprägten Regionen - aus. ${ }^{14}$

Falters zentrale Ergebnisse stehen in Einklang mit der Cleavage-Theorie von Seymour Martin Lipset und Stein Rokkan und ihrer empirischen Anwendung auf Deutschland. ${ }^{15}$ Dieser theoretische Ansatz ist historisch-langfristig ausgerichtet und bietet eine Erklärung zur Entstehung und Struktur des modernen Parteienwettbewerbs in Westeuropa. ${ }^{16}$ Der CleavageTheorie zufolge prägen in Deutschland vor allem zwei Konfliktlinien die Muster des Parteienwettbewerbs und des Wählerverhaltens. ${ }^{17}$ Dies ist zum einen der Konflikt zwischen Staat und römisch-katholischer Kirche, der sich im „Kulturkampf“ der 1870er und 1880er Jahre herausgebildet und zu Gründung und Erfolg der Zentrumspartei als Interessenvertretung der Katholiken in Deutschland auf politischer Ebene bis 1933 und auch in der Gründungsphase der Bundesrepublik geführt hat. Die zweite, historisch gesehen jüngere Konfliktlinie ist die zwischen Arbeit und Kapital und somit zwischen Besitzenden einerseits und lohnabhängigen Arbeitern (und Angestellten) andererseits. Aus diesem Konflikt haben sich als Interessenvertreter auf politischer Ebene die Sozialdemokratische Partei Deutschlands (SPD) und - in Folge der Auseinandersetzung um das in Deutschland nach dem Ersten Weltkrieg zu installierende politische System und die Wirtschafts- und Sozialordnung - die

13 Vgl. ebenda, S. 188, 211.

14 Vgl. ebenda, S. 364 - 374; siehe auch Eckhard Jesse, Hitlers Wähler - Falters Standardwerk, in: ZParl, 24. Jg. (1993), H. 3, S. $533-536$.

15 Vgl. Seymour M. Lipset / Stein Rokkan, Cleavage Structures, Party Systems and Voter Alignments: An Introduction, in: dies. (Hrsg.), Party Systems and Voter Alignments: Cross-National Perspectives, New York / London 1967, S. 1 - 64; siehe auch Franz U. Pappi, Die konfessionell-religiöse Konfliktlinie in der deutschen Wählerschaft: Entstehung, Stabilität und Wandel, in: Dieter Oberndörfer / Hans Rattinger / Karl Schmitt (Hrsg.), Wirtschaftlicher Wandel, religiöser Wandel und Wertewandel, Berlin 1985, S. 269 - 290; Franz U. Pappi / Susumu Shikano, Die politisierte Sozialstruktur als mittelfristig stabile Basis einer deutschen Normalwahl, in: Kölner Zeitschrift für Soziologie und Sozialpsychologie, 54. Jg. (2002), H. 3, S. 444 - 475; Franz U. Pappi, Cleavage, in: Dieter Nohlen / Rainer-Olaf Schultze (Hrsg.), Lexikon der Politikwissenschaft, Bd. 4, München 2010, S. $110-112$.

16 Vgl. Daniele Caramani, The Nationalization of Politics. The Formation of National Electorates and Party Systems in Western Europe, Cambridge 2004; Michael Gallagher / Michael Laver I Peter Mair, Representative Government in Modern Europe, New York 2011, S. 278 - 325; William C. Clark / Sona Golder / Matt Golder, Principles of Comparative Politics, Washington 2012, S. $603-644$.

17 Vgl. hierzu Seymour M. Lipset / Stein Rokkan, a.a.O. (Fn. 15), S. 13 - 21; Franz U. Pappi / Susumu Shikano, a.a.O. (Fn. 15), S. 449 f. 
Kommunistische Partei Deutschlands (KPD) gegründet. ${ }^{18}$ Sowohl die katholische Zentrumspartei als auch die beiden die Interessen der Arbeiterschaft bedienenden Parteien SPD und KPD werden dem „organisationszentrierten Milieu“ als Gegensatz zum „personenzentrierten Milieu“ zugerechnet. ${ }^{19}$ Letzteres ist durch einen schwach ausgeprägten Ausbau der Organisationsnetzwerke und die Relevanz lokaler Honoratioren gekennzeichnet. ${ }^{20}$ Sozialistisches ${ }^{21}$ wie katholisches Milieu sind demnach dadurch charakterisiert, dass sie aus Verfolgung und Bekämpfung durch die Eliten des Kaiserreichs entstanden sind und - in Form der Gewerkschaften und der römisch-katholischen Kirche - stark ausgebaute Organisationsnetzwerke besaßen, die stabilisierend auf das jeweilige Milieu eingewirkt haben. Das katholische Milieu - maßgeblich etabliert durch den „Kulturkampf“ - war tief verankert in der katholischen Kirche und auf politischer Ebene durch die Zentrumspartei vertreten. ${ }^{22}$

Folgt man zudem der Literatur, in der die Persistenz der „sozial-moralischen Milieus“23 hervorgehoben wird, um die Stabilität der Gesellschaftsordnung und insbesondere der Wahlergebnisse im Verlauf von Kaiserreich und Weimarer Republik zu erklären, dann ist für die Reichstagswahl im November 1933, bei der Wähler ihre ablehnende Haltung gegenüber der nationalsozialistischen Diktatur nur durch die Abgabe eines ungültigen Stimmzettels oder auch - indirekt und damit weniger expressiv - durch Nichtwahl ${ }^{24}$ signalisieren konnten, eine hohe Ablehnung insbesondere in Städten und Landkreisen mit einem hohem Katholiken- beziehungsweise hohem Industriearbeiteranteil zu erwarten. Vor dem Hintergrund, dass „sozial-moralische Milieus“ der Studie von M. Rainer Lepsius zufolge durch ein Zusammenkommen mehrerer Strukturdimensionen wie Religion, regionale Tradition, wirtschaftliche Lage oder subjektiv empfundene Schichtzugehörigkeit gebildet werden $^{25}$ und Rohe zudem argumentiert, dass Angehörige unterschiedlicher „sozial-morali-

18 Vgl. Marc Debus / Martin E. Hansen, Die Dimensionalität der Reichstage der Weimarer Republik von 1920 bis 1932, in: PVS, 51. Jg. (2010), H. 1, S. 15 - 42, S. 22.

19 Wolfgang Pyta, Politische Kultur und Wahlen in der Weimarer Republik, in: Gerhard A. Ritter (Hrsg.), Wahlen und Wahlkämpfe in Deutschland. Von den Anfängen im 19. Jahrhundert bis zur Bundesrepublik Deutschland, Düsseldorf 1997, S. 197 - 239.

20 Vgl. ebenda, S. $208 \mathrm{f}$.

21 Pyta identifiziert zusätzlich zum sozialistischen ein kommunistisches Milieu, das sich gegen Ende der 1920er Jahre herausgebildet habe; vgl. ebenda, S. 208.

22 Vgl. Peter Lösche, Kleine Geschichte der deutschen Parteien, Stuttgart 1994, S. 53 f., S. 79 f.; Marc Debus / Martin E. Hansen, a.a.O. (Fn. 18), S. 21.

23 M. Rainer Lepsius, Parteiensystem und Sozialstruktur: Zum Problem der Demokratisierung der deutschen Gesellschaft, in: ders., Demokratie in Deutschland, Göttingen 1993, S. 25 - 50.

24 Die Nichtteilnahme an einer Wahl oder Abstimmung kann natürlich auch durch Gründe wie Krankheit oder Abwesenheit am Wahltag bedingt sein. Hält man sich jedoch vor Augen, dass autoritäre Regime oder Diktaturen - und damit auch das NS-Regime - sowohl eine hohe Zustimmung als auch eine hohe Beteiligung bei von ihnen durchgeführten Wahlen oder Abstimmungen wünschen, um ihre Herrschaft - nachträglich - durch das Volk „eindrucksvoll“ legitimieren zu lassen, und dass - im Fall Deutschlands im November 1933 - die paramilitärischen Truppen des NS-Regimes an manchen Orten Fahrdienste einrichteten, um Bürger an die Wahlurne zu bringen, oder bislang noch nicht zur Wahl gegangene Bürger direkt aufforderten, wählen zu gehen, dann kann unter diesen Umständen der Anteil der Nichtwähler durchaus Ausdruck von Systemopposition sein. Vgl. Jennifer Gandhi / Ellen Lust-Okar, Elections under Authoritarianism, in: Annual Review of Political Science, 12. Jg. (2009), S. 403 - 422, S. 409; Nico Voigtlaender / Hans-Joachim Voth, a.a.O. (Fn. 9), S. 10; siehe auch Richard J. Evans, The Third Reich in Power: 1933-1939, London 2005, S. 108 ff.; Hans-Ulrich Wehler, a.a.O. (Fn. 7), S. 72.

25 Vgl. M. Rainer Lepsius, a.a.O. (Fn. 23), S. 37 f. 
scher" Milieus nicht nur eine gleiche Denk- und Deutungsweise des Alltags haben, sondern auch tatsächlich anders leben ${ }^{26}$, dann dürften sich die beiden organisationszentrierten Milieus nicht direkt mit Beginn der Diktatur im Frühjahr 1933 aufgelöst haben. Vielmehr sollte sich die regionale beziehungsweise lokale Stärke des sozialistischen wie katholischen Milieus noch auf das Wählerverhalten bei der Reichstagswahl im November 1933 und damit auf das Ausmaß der Systemunterstützung für die Diktatur der NSDAP ausgewirkt haben. Da die Reichstagswahl vom November 1933 nur etwas mehr als ein halbes Jahr nach der Aushebelung der freiheitlich-demokratischen Elemente der Weimarer Reichsverfassung durch die Nationalsozialisten stattfand, ist die Erwartung plausibel, dass die Netzwerke des sozialistischen wie katholischen und damit der beiden organisationszentrierten Milieus noch zu einem gewissen Grad Bestand hatten und somit deren lokale Bedeutung am Grad der Wahlbeteiligung und dem Anteil ungültiger, der NSDAP-Liste nicht zustimmender Stimmen sichtbar werden sollte: Je höher der Anteil der sozialen Gruppen, die das sozialistische beziehungsweise katholische Milieu repräsentierten, in der Bevölkerung auf Stadtund Landkreisebene war, desto höher sollte der Anteil der Nichtwähler sowie der ungültigen Stimmen in der jeweiligen Gebietskörperschaft gewesen sein $(H 1)$.

Diese erste Erwartung lässt sich weiter spezifizieren, indem berücksichtigt wird, dass lediglich die Sozialdemokraten aktiv Widerstand - auch auf parlamentarischer Ebene - gegen die Machtübernahme der NSDAP leisteten. So waren die anwesenden Vertreter der SPD die einzigen Abgeordneten im Reichstag, die am 23. März 1933 gegen das einen Tag später in Kraft tretende so genannte „Gesetz zur Behebung der Not von Volk und Reich“ stimmten, während die Mitglieder aller anderen Fraktionen und damit auch die Abgeordneten des katholischen Zentrums dafür votierten. Gerade das Abstimmungsverhalten der Zentrumsfraktion sowie der Vertreter der Bayerischen Volkspartei (BVP) ermöglichte - zusammen mit der Änderung der Geschäftsordnung des Reichstages, derzufolge die aufgrund von Verhaftung oder Flucht fehlenden 81 KPD- und 26 SPD-Abgeordneten als ,unentschuldigt fehlend" und damit als anwesend gerechnet wurden ${ }^{27}$ - das Erringen einer Zweidrittelmehrheit der anwesenden Abgeordneten für das Ermächtigungsgesetz. Zwar gab es innerhalb von Zentrum und auch BVP massive Auseinandersetzungen über die Frage von Zustimmung und Ablehnung, jedoch setzten sich - auch aufgrund der Versprechungen Hitlers, die Rechte insbesondere der römisch-katholischen Kirche zu respektieren - die Befürworter einer nationalen Sammlungsbewegung durch, so dass alle anwesenden Zentrumsund BVP-Fraktionsmitglieder für das Gesetz stimmten. ${ }^{28}$ Schließlich hatte die Deutsche Bischofskonferenz bereits im März 1933 alle Deutschen zur Loyalität gegenüber der Regierung von Hitler aufgerufen, und Reichstagsabgeordnete von DNVP wie auch des Zentrums versuchten nach den Selbstauflösungen ihrer jeweiligen Partei in der zweiten Juni- und ersten Juli-Hälfte 1933, als Hospitanten in die Fraktion der NSDAP im Reichstag aufgenommen zu werden. ${ }^{29}$ Da zudem auf der NSDAP-Liste zur Reichstagswahl am 12. November 1933 - sicherlich auch zur Wahrung des Scheins der Überparteilichkeit - frühere Politiker des Zentrums sowie der BVP und damit Repräsentanten des katholischen Milieus

26 Vgl. Karl Rohe, a.a.O. (Fn. 11), S. 19 f.

27 Vgl. Ulrich Thamer, Der Nationalsozialismus, Stuttgart 2002, S. 136.

28 Vgl. ebenda, S. $136 \mathrm{ff}$.

29 Vgl. Hans-Ulrich Wehler, a.a.O. (Fn. 7), S. $67-71$. 
kandidierten ${ }^{30}$, kann davon ausgegangen werden, dass der Widerstand der Katholiken gegen die NS-Diktatur und damit gegen die NSDAP-Liste zur Reichstagswahl vom November 1933 schwächer ausgeprägt war als seitens des sozialistischen Milieus, weil sich große Teile des katholischen Milieus mit dem durch die Nationalsozialisten errichteten Regime arrangiert hatten. Im Hinblick auf die Determinanten des Wählerverhaltens bei der Reichstagswahl vom 12. November 1933 kann daher die Hypothese formuliert werden, dass weniger die Dominanz des katholischen als vielmehr des sozialistischen Milieus in der Bevölkerung den Anteil der Nichtwähler sowie der ungültigen Stimmen in den jeweiligen Gebietskörperschaften positiv beeinflusste (H2).

Hinzu kommt, dass die Überwachung der Bürgerschaft und der Wähler - auch mittels Mechanismen der sozialen Kontrolle - durch die staatlichen Institutionen in einem autoritären System in ländlichen Regionen leichter durchzuführen sein dürfte als in urbanen Gegenden, mit entsprechenden Folgen für die Wahlbeteiligung und das Wählerverhalten. ${ }^{31}$ Demzufolge ist zu erwarten, dass der Anteil der Nichtwähler sowie derjenigen Wähler, die durch die Abgabe eines ungültigen Stimmzettels nicht für die NSDAP-Liste stimmten, in Städten sowie in Landkreisen mit großer Bevölkerungszahl höher ausfielen als in ländlichdörflichen Regionen (H3).

Zum Testen dieser aus dem Stand der Forschung zu Politischer Kultur, Partizipation und Wählerverhalten in der Weimarer Republik abgeleiteten Hypothesen bedarf es solchen Datenmaterials, das eine genügend hohe Anzahl an Fällen umfasst, so dass multivariate Regressionsanalysen möglich sind. Letztere erlauben die Ermittlung des Effekts einer erklärenden Variablen unter wechselseitiger Kontrolle der übrigen, theoretisch hergeleiteten unabhängigen Variablen auf die Ausprägungen des zu erklärenden Phänomens.

\section{Daten und methodisches Vorgehen}

Die Ergebnisse der Reichstagswahl vom 12. November 1933 auf Ebene der Stadt- und Landkreise sind - nebst der Kandidatenliste und einer Kopie eines Stimmzettels - in Band 449 der „Statistik des Deutschen Reiches“ abgedruckt. ${ }^{32}$ Der Band gibt unter anderem die

30 Beispielhaft können der BVP-Politiker Eugen von Quadt zu Wykradt und Isny, Albert Hackelsberger, der Anfang 1933 zum stellvertretenden Vorsitzenden des Zentrums gewählt wurde, oder Oskar Farny, der von 1930 bis Juni 1933 für das Zentrum Mitglied des Reichstages und 1953 kurzzeitig Mitglied der CDU/CSU-Bundestagsfraktion war, um dann bis 1960 als Minister in die baden-württembergische Landesregierung zu wechseln, angeführt werden. Vgl. Frank Raberg, Oskar Farny - Ein bewährter Demokrat?, in: Wolfgang Proske (Hrsg.), Täter Helfer Trittbrettfahrer, Bd. 4: NS-Belastete aus Oberschwaben, Gerstetten 2015, S. $114-127$.

31 Vgl. Jennifer Gandhi / Ellen Lust-Okar, a.a.O. (Fn. 24), S. 409. Zur Analyse der Wahlbeteiligung in unterschiedlichen lokalen Kontexten siehe auch Armin Schäfer, Beeinflusst die sinkende Wahlbeteiligung das Wahlergebnis? Eine Analyse kleinräumiger Wahldaten in deutschen Großstädten, in: PVS, 53. Jg. (2012), H. 2, S. 240 - 264; ders. / Sigrid Roßteutscher, Räumliche Unterschiede der Wahlbeteiligung bei der Bundestagswahl 2013: Die soziale Topografie der Nichtwahl, in: Karl-Rudolf Korte (Hrsg.), Die Bundestagswahl 2013: Analysen der Wahl-, Parteien-, Kommunikations- und Regierungsforschung, Wiesbaden 2014, S. 99 - 118; Edward Fieldhouse / David Cutts / Peter John / Paul Widdop, When Context Matters: Assessing Geographical Heterogeneity of Get-out-the-vote Treatment Effects Using a Population based Field Experiment, in: Political Behavior, 36. Jg. (2014), H. 1, S. 77 - 97.

32 Vgl. Statistisches Reichsamt, a.a.O. (Fn. 8), S. 8 - 105. 
Resultate für alle Land- und Stadtkreise ${ }^{33}$ sowie derjenigen Gemeinden innerhalb der Landkreise wieder, die mehr als 2.000 Einwohner hatten. ${ }^{34}$ Herangezogen werden hier im Gegensatz zur Studie von Falter ${ }^{35}$ - jedoch nur die Ergebnisse auf Ebene der Land- und Stadtkreise. Die Anzahl der Stimmberechtigten, die der abgegebenen Stimmen, der ungültigen Stimmen und der Stimmen für den „Kreiswahlvorschlag der NSDAP“ ermöglichen es, den Anteil der ungültigen Stimmen an allen abgegebenen Stimmen zu berechnen, was als ein Indikator für die direkt an der Wahlurne geäußerte Ablehnung gegenüber der Diktatur der NSDAP angesehen werden kann. Zudem kann ein weiterer Indikator für direkt wie indirekt geäußerte Opposition gegenüber dem Regime gebildet werden, der sich aus dem Anteil der ungültigen Stimmen und dem der Nichtwähler an allen Wahlberechtigten zusammensetzt. Hans-Ulrich Wehler zufolge fanden bei der Reichstagswahl vom November 1933 keine systematischen Wahlmanipulationen statt, so dass die von der offiziellen Statistik ausgewiesenen Daten als weitgehend fälschungsfrei angesehen werden können. ${ }^{36}$ Jedoch gab es seitens des NS-Regimes und seiner paramilitärischen Einheiten direkten wie indirekten Druck auf die Bürgerschaft, sich an der Wahl zu beteiligen und im Sinne der Machthaber abzustimmen. ${ }^{37}$ Die Höhe der Wahlbeteiligung kann daher auch als ein Indikator für die Zustimmung zu beziehungsweise die Zufriedenheit mit einem politischen System gerade in autoritären Systemen angesehen werden. ${ }^{38}$ Wenn keine Möglichkeit gegeben ist, gegen die Regierung zu stimmen, und somit kein Alternativangebot gemacht wird beziehungsweise werden darf, ist Nichtwahl eine Möglichkeit, wie Bürger ihre Unzufriedenheit, ihren Protest oder ihre Ablehnung eines politischen Systems ausdrücken können. ${ }^{39}$ Daher sind Machthaber in autoritären Regimen in der Regel an hohen Beteiligungsraten bei Abstimmungen und Wahlen, die jeweils ohne echten Wettbewerb stattfinden, interessiert, um so nach innen wie nach außen die - vermeintliche - Zufriedenheit der Bevölkerung mit dem Status quo zu signalisieren. ${ }^{40}$

Die Ausprägungen dieser beiden abhängigen Variablen in den deutschen Stadt- und Landkreisen bei der Reichstagswahl vom 12. November 1933 sollen mit Variablen erklärt werden, die als Indikatoren für die Stärke des sozialistischen wie katholischen Milieus wie auch für das Ausmaß sozialer beziehungsweise politischer Kontrolle dienen können. In Anlehnung an die Ergebnisse der Studie von Falter und an die Literatur zu den Eigenschaften der sozial-moralischen Milieus in der Weimarer Republik werden dazu der Anteil der Industriearbeiterschaft sowie der Katholiken an der Bevölkerung in den Städten und Landkreisen als Kernindikatoren für die regionale Präsenz und Ausprägung des sozialistischen und katholi-

33 In den Ländern des Deutschen Reiches gab es keine einheitliche Bezeichnung für Landkreise wie für kreisfreie Städte. Der Einfachheit halber wird hier die Bezeichnung „Landkreis“ und „Stadtkreis" gewählt.

34 Vgl. Statistisches Reichsamt, a.a.O. (Fn. 8), S. 3.

35 Vgl. Jürgen W. Falter, a.a.O. (Fn. 2), S. $54-66$.

36 Vgl. Hans-Ulrich Wehler, a.a.O. (Fn. 7), S. 72.

37 Vgl. Nico Voigtlaender / Hans-Joachim Voth, a.a.O. (Fn. 9), S. 10; Richard J. Evans, a.a.O. (Fn. 24), S. $108 \mathrm{ff}$.

38 Vgl. etwa Albert O. Hirschmann, Exit, Voice, and Loyalty: Responses to Decline in Firms, Organizations and States, Cambridge 1970; Pippa Norris, Critical Citizens: Global Support for Democratic Government: Global Support for Democratic Government, Oxford 1999.

39 Vgl. zum Beispiel Dominik Allenspach, Der Effekt der Systemunterstützung auf die politische Partizipation. Eine vergleichende Analyse westlicher Demokratien, Wiesbaden 2012.

40 Vgl. Jennifer Gandhi / Ellen Lust-Okar, a.a.O. (Fn. 24). 
schen Milieus herangezogen. ${ }^{41}$ Die Daten liegen für das Jahr 1925 auf der Grundlage der zu diesem Zeitpunkt durchgeführten Volkszählung vor und sind dem Datensatz von Falter und Hänisch ${ }^{42}$ entnommen. Gleiches gilt für die - sehr ungleich über die 895 Land- und Stadtkreise, die aufgrund der Datenverfügbarkeit in der Analyse berücksichtigt werden können, verteilte - Anzahl der Bevölkerung in den Städten- und Landkreisen sowie für den Anteil der Einwohner jüdischen Glaubens. Zur Kontrolle wird zusätzlich eine dichotom codierte Variable in die Analyse integriert, die zwischen Landkreisen und Städten differenziert. Der Datensatz von Falter und Hänisch, der den Informationen zu den Wahlergebnissen auf Ebene der Land- und Stadtkreise bei der Reichstagswahl vom 12. November 1933 zugespielt wurde, ist um weitere Informationen ergänzt worden, die unter anderem die Wahlergebnisse der Reichstagswahl vom 5. März 1933 umfassen, so dass als weitere Kontrollvariablen der Anteil ungültiger Stimmen an allen abgegebenen Stimmen sowie der Anteil von Nichtwählern und ungültigen Stimmen an allen Wahlberechtigten je Gebietskörperschaft bei der Reichstagswahl vom März 1933 in die Analyse - quasi als zeitverzögerte abhängige Variable („lagged dependent variable“) - einbezogen werden können.

Die hier angewandte Methode zur Bestimmung der ausschlaggebenden Faktoren für die Höhe des Anteils der ungültigen Stimmen an allen abgegebenen Stimmen und für die Summe des Nichtwähleranteils und der ungültigen Stimmen an allen Wahlberechtigten in den Stadt- und Landkreisen ist eine Tobit-Regression, da die beiden abhängigen Variablen jeweils nach unten und oben begrenzt sind und theoretisch nicht unter einem Wert von null Prozent beziehungsweise über einem Wert von 100 Prozent liegen können. Um weitere kontextuelle Faktoren zu kontrollieren, die nicht durch die bereits in die empirischen Modelle integrierten Variablen abgedeckt werden und die sich beispielsweise auch aus der unterschiedlichen Größe der Gebietskörperschaften ergeben können ${ }^{43}$, werden die Standardfehler für die Land- und Stadtkreise geclustert. Dies erlaubt eine konservativere Schätzung der Determinanten des Anteils ungültiger Stimmen an allen abgegebenen Stimmen beziehungsweise von Nichtwählern und ungültigen Stimmen an allen Wahlberechtigten.

\section{Ergebnisse}

Die in Abbildung 1 dargestellten Boxplots verdeutlichen die Varianz auf den beiden abhängigen Variablen. Zwar weisen mehr als die Hälfte aller Land- und Stadtkreise einen Anteil von weniger als zehn Prozent ungültiger Stimmen auf. Dennoch gibt es Fälle, in denen die

41 Vgl. Jürgen W. Falter, a.a.O. (Fn. 2), S. 198 ff.; M. Rainer Lepsius, a.a.O. (Fn. 23), S. 33 ff., S. 47 ff.; Wolfgang Pyta, a.a.O. (Fn. 19), S. 222.

42 Vgl. Jürgen W. Falter / Dirk Hänisch, Election and Social Data of the Districts and Municipalities of the German Empire from 1920 to 1933. GESIS Datenarchiv, Köln 1990 (ZA8013, Version 1.0.0, doi:10.4232/1.8013). Der bei GESIS hinterlegte Datensatz ZA8013 umfasst für das Jahr 1933 leider nur Informationen über die Berufsstruktur auf Ebene der Städte und Landkreise, nicht jedoch zur Konfessionsstruktur, so dass - um die Ausprägungen der zentralen unabhängigen Variablen zu einem Zeitpunkt zu erfassen - auf die Werte aus dem Jahr 1925 in der Analyse zurückgegriffen werden muss. Vgl. Dirk Hänisch, Inhalt und Struktur der Datenbank „Wahl- und Sozialdaten der Kreise und Gemeinden des Deutschen Reiches von 1920 bis 1933“, in: Historical Social Research / Historische Sozialforschung, 14. Jg. (1989), H. 1, S. 39 - 67, S. 42.

43 Siehe Jürgen W. Falter, a.a.O. (Fn. 2), S. 61 f. 


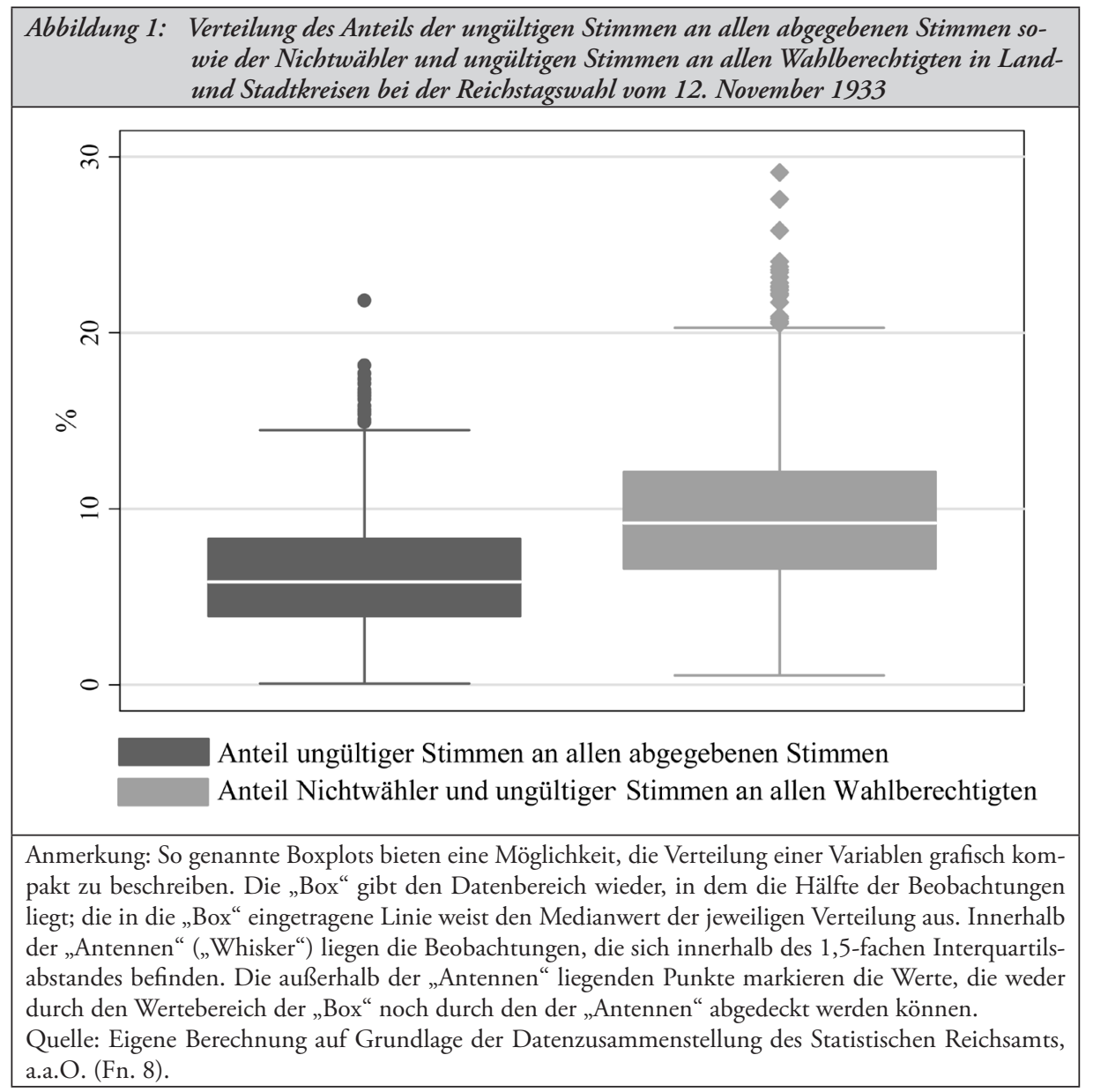

direkte Ablehnung der NSDAP-Liste durch die Abgabe einer ungültigen Stimme bei über 15 Prozent, teilweise bei über 20 Prozent lag. Ein ähnliches Bild ergibt sich, wenn man die Verteilung der Variable betrachtet, die den Anteil der Nichtwähler und ungültigen Stimmen an den Walberechtigten in den Land- und Stadtkreisen umfasst. In rund der Hälfte der Gebietskörperschaften variiert der Wert zwischen sechs und zwölf Prozent; allerdings gab es Landkreise und Städte, wo sich am 12. November 1933 bis zu knapp 30 Prozent der Wahlberechtigten entweder nicht an der Abstimmung beteiligten oder die einzig zur Wahl stehende Kandidatenliste durch eine ungültige Stimmabgabe ablehnten. Dies sind für Diktaturen oder andere, nicht-monarchisch geprägte autoritäre Regime durchaus hohe Werte.

Lassen sich die in Abbildung 1 grafisch dargestellten und in der Einleitung zu diesem Beitrag exemplarisch dargestellten Unterschiede im Ausmaß der direkt wie indirekt bei der Reichstagswahl vom 12. November 1933 geäußerten Ablehnung der NSDAP-Liste mit den herangezogenen Indikatoren erklären? Zeigen sich Unterschiede in der Erklärungskraft der Variablen, die Informationen zur regionalen Verankerung des sozialistischen sowie des katholischen Milieus umfassen? Tabelle 1 weist die Ergebnisse der Tobit-Regressionsanalysen 
aus. Während beim ersten Modell der Anteil der ungültigen Stimmen an allen abgegebenen Stimmen die abhängige Variable darstellt, wird im zweiten Modell - auch vor dem Hintergrund der großen Bedeutung hoher Beteiligungswerte bei Wahlen und Abstimmungen in autoritären Regimen und Diktaturen - der aufsummierte Anteil von Nichtwählern und ungültigen Stimmen an allen Wahlberechtigten als zu erklärende Variable herangezogen. In beiden Modellen weisen der Anteil der Industriearbeiter an der Bevölkerung, die Einwohnerzahl, die Stadtkreis-Dummyvariable sowie der Anteil ungültiger Stimmen beziehungsweise der aufsummierte Anteil von Nichtwählern und ungültigen Stimmen dieselben Effekte auf, die zudem auf den gängigen Interpretationsniveaus statistisch signifikant sind. ${ }^{44}$ Je höher der Anteil der Industriearbeiter in einem Land- beziehungsweise Stadtkreis war, desto höher fiel - unter Kontrolle aller weiteren in die Analyse einbezogenen unabhängigen Variablen - sowohl der Anteil der ungültigen Stimmen an allen abgegebenen Stimmen als auch der Anteil der Nichtwähler und ungültig Wählenden an allen Wahlberechtigten im November 1933 aus. Dies deutet darauf hin, dass mit stärkerer Ausprägung des sozialistischen Milieus auf Ebene der Land- und Stadtkreise die direkt wie indirekt geäußerte Ablehnung des NS-Regimes bei der Reichstagswahl im Spätherbst 1933 zunahm. Gleiches galt mit höherer Einwohnerzahl sowie in Städten im Vergleich zu den Landkreisen: Der Anteil ungültiger abgegebener Stimmen sowie der Anteil von Nichtwählern und ungültigen Stimmen an den Wahlberechtigten stieg mit wachsender Einwohnerzahl beziehungsweise war in Städten signifikant höher als in Landkreisen. Dies bestätigt die dritte sowie - zumindest im Hinblick auf den Industriearbeiteranteil - die erste Hypothese.

Der Anteil der Bevölkerung jüdischen Glaubens, der als Kontrollvariable in die Regressionsanalyse eingebaut wurde, weist im ersten Modell keinen signifikanten Effekt auf, ist hingegen positiv und signifikant in Modell 2: Je größer der prozentuale Anteil der Juden an der Gesamtbevölkerung - gemessen anhand der Volkszählung 1925 - war, desto höher fiel in den jeweiligen Land- und Stadtkreisen der Anteil von Nichtwählern und ungültigen Stimmen an allen Wahlberechtigten aus. Die als „zeitverzögerte abhängige Variablen“ zur weiteren Kontrolle in die Analyse einbezogenen Variablen des Anteils ungültiger Stimmen beziehungsweise des Anteils der Nichtwähler und ungültigen Stimmen an allen Wahlberechtigten hatten - wie erwartet - einen positiven Effekt: dort, wo es bereits im Frühjahr 1933 einen höheren Anteil Nichtwähler und/oder ungültiger Stimmen gab, war dieser auch im November 1933 hoch.

Der Effekt der Variable, die den Anteil der Katholiken auf Stadt- und Landkreisebene wiedergibt, ist hingegen nur im ersten Modell signifikant verschieden von Null und weist dort den - gemäß der ersten Hypothese erwarteten - positiven Effekt auf: Mit zunehmendem Anteil an römisch-katholischer Bevölkerung stieg der Anteil der ungültigen und damit die

$44 \mathrm{Da}$ in den Sozialwissenschaften meist mit Näherungsindikatoren gearbeitet werden muss, die ein bestimmtes theoretisches Konzept reflektieren, und die so herangezogenen Variablen bei Weitem nicht immer genau das widerspiegeln beziehungsweise widerspiegeln können, was gemäß der jeweiligen Theorie eigentlich gemessen werden soll, ist es auch bei Analysen, die auf Vollerhebungen basieren, angebracht, Standardfehler und Signifikanzniveaus im Rahmen der Interpretation der Ergebnisse zu berücksichtigen. Auf diese Weise kann dazu beigetragen werden, dass auf der Grundlage der Ergebnisse der statistischen Schätzung Schlussfolgerungen gezogen werden, die auch gegenüber etwaigen Schwankungen oder Messfehlern in den ausgewählten Daten robust sind. Vgl. hierzu Andreas Broscheid / Thomas Gschwend, Zur statistischen Analyse von Vollerhebungen, in: PVS, 46. Jg (2005), H. 1, S. $16-26$. 
NSDAP-Liste ablehnenden Stimmen an allen abgegebenen Stimmen tendenziell an. Dies gilt jedoch nicht für den aufsummierten Anteil von Nichtwählern und ungültigen Stimmen an allen Wahlberechtigten: Der Effekt dieser Variable ist - auf den gängigen statistischen Signifikanzniveaus - nicht als verschieden von Null zu interpretieren (vgl. Tabelle 1). Dieses Ergebnis spricht somit nicht für die erste, sondern vielmehr für die zweite Hypothese; diese ging aufgrund der Repräsentation ehemaliger BVP- und Zentrumsfunktionäre und -mitglieder auf der NSDAP-Liste, des erfolgreichen Abschlusses der Reichkonkordatsverhandlungen mit der katholischen Kirche am 20. Juli 1933 sowie der Empfehlung der Fuldaer Bischofskonferenz vom 28. März 1933, loyal gegenüber dem neuen, autoritären System zu agieren ${ }^{45}$, nicht davon aus, dass eine stärkere regionale Präsenz des katholischen Milieus zu einer höheren direkten wie indirekten Ablehnung des Nationalsozialismus bei der Reichstagswahl am 12. November 1933 führen sollte.

\begin{tabular}{|c|c|c|c|c|}
\hline \multicolumn{5}{|c|}{$\begin{array}{l}\text { Tabelle 1: Determinanten des Anteils ungültiger Stimmen an allen abgegebenen Stimmen } \\
\text { (Modell 1) sowie von Nichtwählern und ungültigen Stimmen an allen Wablbe- } \\
\text { rechtigten (Modell 2) bei der Reichstagswabl am 12. November } 1933\end{array}$} \\
\hline & \multicolumn{2}{|c|}{ Modell 1} & \multicolumn{2}{|c|}{ Modell 2} \\
\hline Anteil der Industriearbeiter (1925) & $0.08^{* *}$ & $(0.01)$ & $0.09^{* *}$ & $(0.01)$ \\
\hline Anteil der Katholiken (1925) & $0.01^{*}$ & $(0.00)$ & -0.00 & $(0.00)$ \\
\hline Anteil der Juden (1925) & -0.18 & $(0.13)$ & $0.42^{+}$ & $(0.22)$ \\
\hline Bevölkerung (in Tausend, 1933) & $0.01^{* *}$ & $(0.00)$ & $0.01^{* *}$ & $(0.00)$ \\
\hline Stadtkreis & $2.85^{* *}$ & $(0.28)$ & $4.20^{* *}$ & $(0.35)$ \\
\hline Anteil ungültiger Stimmen, Reichstagswahl März 1933 & $0.66^{* *}$ & $(0.25)$ & & \\
\hline $\begin{array}{l}\text { Anteil von Nichtwählern und ungültigen Stimmen, } \\
\text { Reichstagswahl März } 1933\end{array}$ & & & $0.17^{* *}$ & $(0.03)$ \\
\hline Konstante & $2.36^{* *}$ & $(0.25)$ & $3.63^{* *}$ & $(0.49)$ \\
\hline Sigma & $2.61^{* *}$ & $(0.08)$ & $3.40^{* *}$ & $(0.10)$ \\
\hline $\mathrm{N}$ & \multicolumn{2}{|c|}{895} & \multicolumn{2}{|c|}{895} \\
\hline pseudo $\mathrm{R}^{2}$ & \multicolumn{2}{|c|}{0.098} & \multicolumn{2}{|c|}{0.092} \\
\hline AIC & \multicolumn{2}{|c|}{4271.48} & \multicolumn{2}{|c|}{4746.34} \\
\hline \multicolumn{5}{|c|}{$\begin{array}{l}\text { Anmerkungen: Ergebnisse einer Tobit-Regressionsanalyse mit nach Land- und Stadtkreisen geclusterten } \\
\text { Standardfehlern in Klammern. Signifikanzniveaus: }+=\mathrm{p}<0.1{ }^{*}=\mathrm{p}<0.05 ;{ }^{* *}=\mathrm{p}<0.01 \text {. } \\
\text { Quelle: Eigene Berechnung auf Grundlage der Datenzusammenstellung des Statistischen Reichsamts, } \\
\text { a.a.O. (Fn. 8). }\end{array}$} \\
\hline
\end{tabular}

Betrachtet man grafisch die substantiellen Effekte der Variablen, die als Indikatoren für die Stärke des sozialistischen wie katholischen Milieus auf Landkreis- und Stadtebene herangezogen wurden, kann die zweite Hypothese weiter untermauert werden. Wird auf der Grundlage der Regressionsergebnisse der erwartete Anteil an ungültigen Stimmen an allen abgegebenen Stimmen von Nichtwählern und ungültigen Stimmen an den Wahlberechtigten in Abhängigkeit des Anteils von Industriearbeitern beziehungsweise Katholiken in der Bevölkerung in den Land- und Stadtkreisen ermittelt, zeigen sich sehr deutliche Unterschiede. Während in einem Landkreis oder einer Stadt mit sehr geringem Industriearbeiter-

45 Vgl. Hans-Ulrich Wehler, a.a.O. (Fn. 7), S. 63, S. 67; Ulrich Thamer, a.a.O. (Fn. 27), S. 134 138, S. 147. 


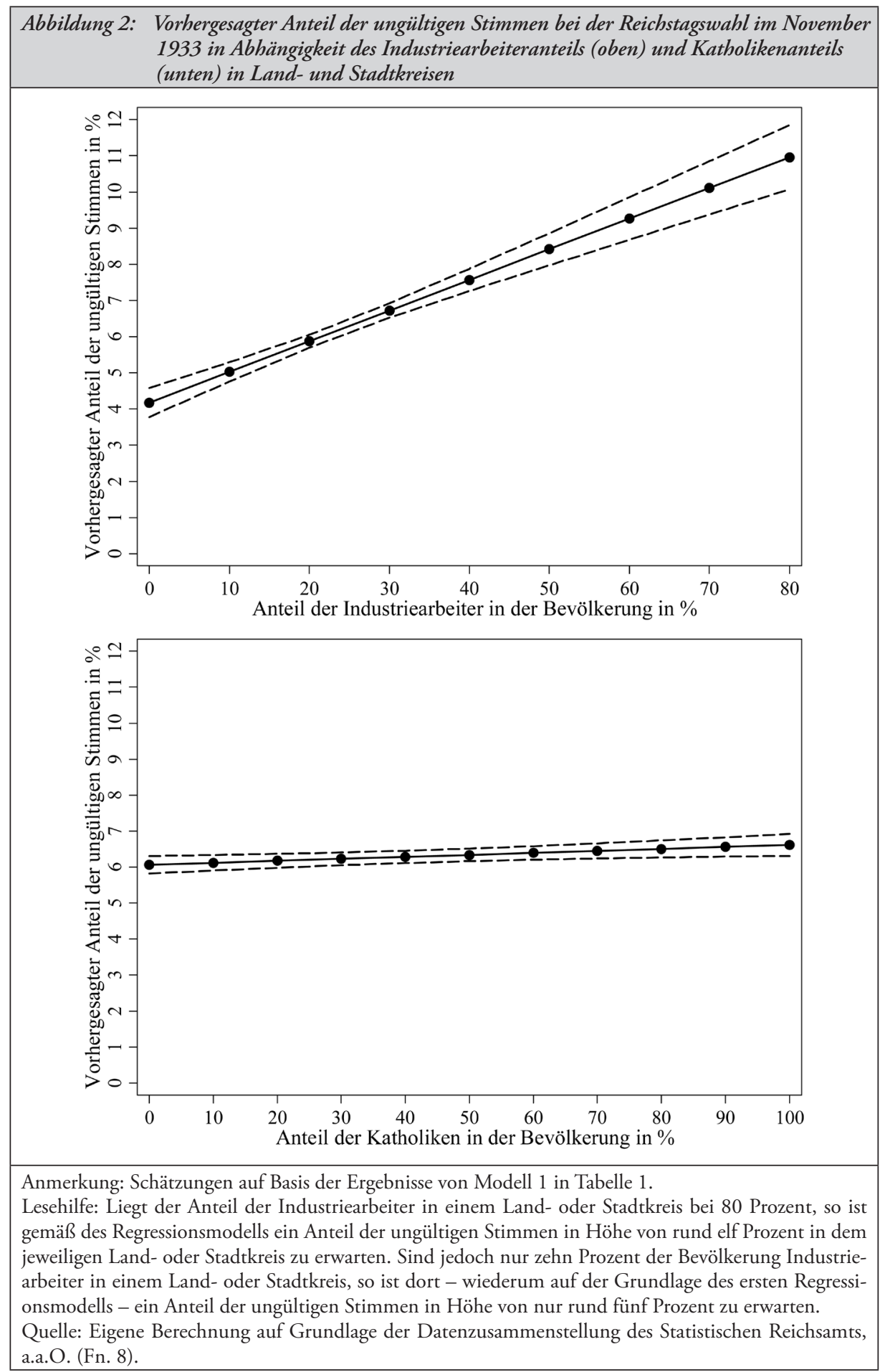


anteil - beispielsweise von unter fünf Prozent - der auf der Basis des ersten Regressionsmodells vorhergesagte Anteil der ungültigen Stimmen zwischen vier und fünf Prozent liegen sollte, so ergibt sich ein geschätzter Wert zwischen zehn und zwölf Prozent in den Gebietskörperschaften, die durch einen sehr hohen Anteil an Industriearbeitern geprägt waren (vgl.
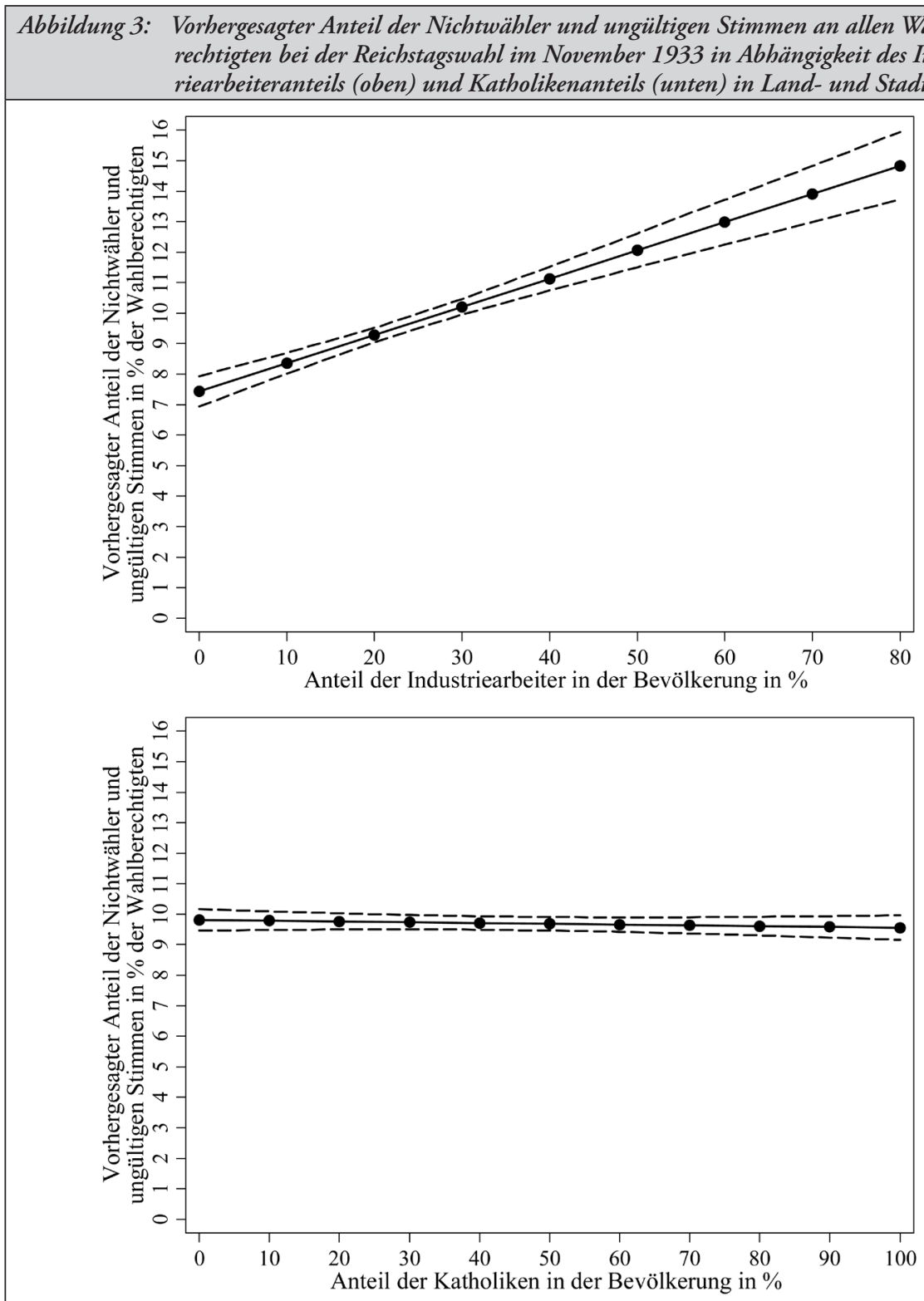

Anmerkung: Schätzungen auf Basis der Ergebnisse von Modell 2 in Tabelle 1.

Lesehilfe: Siehe Abbildung 2.

Quelle: Eigene Berechnung auf Grundlage der Datenzusammenstellung des Statistischen Reichsamts, a.a.O. (Fn. 8). 
Abbildung 2). Im Gegensatz dazu variiert der von Modell 1 vorhergesagte Anteil ungültiger Stimmen an allen abgegebenen Stimmen in Abhängigkeit des Katholikenanteils in der Bevölkerung kaum und liegt nahezu kontinuierlich zwischen sechs und sieben Prozent.

Dasselbe, im Hinblick auf den Effekt des Anteils der katholischen Bevölkerung in den Land- und Stadtkreisen noch stärker ausgeprägte Bild ergibt sich, wenn die Ergebnisse des zweiten Regressionsmodells aus Tabelle 1 herangezogen werden. Abbildung 3 zeigt sehr deutlich, dass mit zunehmendem Anteil der Industriearbeiter an der Bevölkerung das direkt wie indirekt geäußerte Missfallen am zur Wahl stehenden Parteienangebot statistisch signifikant von 7,4 Prozent (bei einem Anteil von null Prozent Industriearbeiter im jeweiligen Stadt- oder Landkreis) auf 14,8 Prozent (bei einem Anteil von 80 Prozent Industriearbeiter) anstieg. Der Anteil der Katholiken in den Land- und Stadtkreisen beeinflusst die Ausprägungen der abhängigen Variable nicht und liegt, unabhängig davon, wie hoch der Katholikenanteil an der Bevölkerung war, gemäß der Schätzungen des Regressionsmodells nur zwischen 9,5 und 9,8 Prozent. Diese Schätzungen beziehen die Ausprägungen der weiteren in den beiden Regressionsmodellen berücksichtigten unabhängigen Variablen wie die Bevölkerungszahl oder den Status einer Gebietskörperschaft als Stadtkreis mit ein. Dadurch werden diese Faktoren, die unter anderem Indikatoren für eine größere soziale Kontrolle sind und damit indirekt Maße für den Druck auf die Bürger darstellen, zur Wahl zu gehen und regimekonform abzustimmen, bei der Ermittlung der vorhergesagten Werte, die in Abbildung 2 und 3 präsentiert werden, kontrolliert. Auf dieser Grundlage kann damit der jeweils explizit von der Milieustärke ausgehende Effekt in den beiden Abbildungen dargestellt werden.

Die Ergebnisse der Analysen unterstützen somit Hypothesen 2 und 3, nicht jedoch die erste Erwartung, die von einem positiven Effekt der Stärke sowohl des sozialistischen als auch des katholischen Milieus auf den Grad der fehlenden Unterstützung für das NS-Regime ausging - operationalisiert über die Anteile ungültiger Stimmen an den abgegebenen Stimmen sowie der ungültigen Stimmen und Nichtwähler an den Wahlberechtigten in den Land- und Stadtkreisen. Lediglich mit zunehmendem Industriearbeiteranteil in der regionalen Bevölkerung stiegen die Werte der beiden unabhängigen Variablen signifikant an, nicht jedoch mit anwachsendem Katholikenanteil.

\section{Wählerverhalten in der Diktatur: katholisches Milieu verändert Haltung zur NSDAP}

Während die Untersuchung des Parteiensystems, seiner gesellschaftlichen Grundlagen und des Wählerverhaltens zur Zeit der Weimarer Republik Gegenstand zahlreicher Untersuchungen war, stand eine detailliertere Analyse der Reichstagswahl vom 12. November 1933 noch aus. Die mit Hilfe multivariater Analysemethoden gewonnenen Ergebnisse der vorliegenden Studie haben gezeigt, dass vor allem die regionale Stärke des sozialistischen Milieus - messbar über den Anteil der Industriearbeiterschaft an der Bevölkerung - zu einer bei dieser Wahl geringeren Unterstützung des NS-Regimes geführt hat. Letzteres galt jedoch nicht für das katholische Milieu, dessen Mitglieder - auf der Grundlage von Analysen des Wählerverhaltens in der Weimarer Republik ${ }^{46}$ - als weitgehend resistent gegenüber dem Nationalsozialismus galten. Möglicherweise hat sowohl das Reichskonkordat als auch die 
Offenheit des rechten Flügels des Zentrums um dessen von 1928 bis Mai 1933 amtierenden Vorsitzenden, Prälat Ludwig Kaas, dazu geführt, dass - im Gegensatz zu den dem sozialistischen Milieu angehörenden Bevölkerungsgruppen - vom Anteil der katholischen Bevölkerung in einem Land- oder Stadtkreis kein ausschlaggebender Einfluss auf die direkte wie indirekte Ablehnung der NSDAP-Liste bei der Reichstagswahl am 12. November 1933 ausging.

Natürlich ist den hier gewonnenen Erkenntnissen mit einem gewissen Grad an Vorsicht zu begegnen. Dies liegt nicht nur an der Gefahr des ökologischen Fehlschlusses bei der Interpretation der Resultate und damit der Schlussfolgerungen, die aufgrund der Eigenschaften eines Kollektivs auf die Merkmale der - ebenfalls in aggregierter Form vorhandenen - abhängigen Variablen gezogen werden. Schließlich stellt die Untersuchungseinheit in der vorliegenden Analyse nicht ein Individuum dar, das wahlberechtigt war und zur Wahl gegangen ist, sondern vielmehr den Anteil bestimmter Bevölkerungsgruppen in den Landkreisen und Städten Deutschlands, von denen theoriegeleitet vermutet werden kann, dass sie eine hohe Abneigung gegenüber einer Wahl der NSDAP hatten. Auch kann nicht dafür garantiert werden, dass die Angaben in der Datenzusammenstellung des Statistischen Reichsamts, die sich nur auf Landkreise, Stadtkreise und Orte innerhalb der Landkreise mit mehr als 2.000 Einwohner bezieht, vollständig korrekt sind. So könnten - gerade vor dem Hintergrund, dass diese Parlamentswahl zur Zeit der NSDAP-Diktatur mit ausgehebelten demokratischen Rechten stattfand - die Einträge auf Wahlbezirks- beziehungsweise kommunaler Ebene zugunsten der Partei teilweise gefälscht worden sein, so dass die Ausprägungen der abhängigen Variable auf Ebene der Land- und Stadtkreise nicht der Wirklichkeit entsprächen. Da die Datenzusammenstellung des Statistischen Reichsamts keine Informationen auf Wahlbezirksebene ausweist, kann dieser Vermutung leider nicht nachgegangen werden. Jedoch existieren - bei gegebener Datenlage - statistische Verfahren, die systematische Wahlfälschung aufzudecken helfen. ${ }^{47}$ Anzumerken ist auch, dass die Gebietsreformen auf der Kreisebene der Jahre 1932/33 dazu führen können, dass die Übereinstimmung zwischen den Einheiten nicht immer perfekt ist. ${ }^{48}$ Trotz dieser Nachteile birgt die Analyse von - weitgehend ohne massive Manipulationen ablaufenden - Parlamentswahlen in Diktaturen, wie es zumindest die Reichstagswahl vom November 1933 gemäß der Einschätzung von Hans-Ulrich Wehler war ${ }^{49}$, eine Möglichkeit, das Verhalten bestimmter sozialer Gruppen zu analysieren und - je nach Kontext und bei Vorliegen entsprechender Daten - in Bezug zu setzen zu vormaligen Wahlen, die in einem demokratischen Verfahren stattgefunden haben. Mit dem hier verwendeten Datenmaterial konnte gezeigt werden, dass offenbar in weiten Teilen des katholischen Milieus eine Veränderung in Bezug zur Haltung gegenüber der NSDAP nach der Machtübernahme der Nationalsozialisten stattgefunden hat, die es sich lohnt, mit qualitativ ausgerichteten historisch-soziologischen Studien weiter zu beleuchten.

47 Vgl. etwa Susumu Shikano / Verena Mack, When does 2nd Digit Benford's Law-Test Signal an Election Fraud? Facts or Misleading Test Results, in: Journal of Economics and Statistics, 231. Jg. (2011), H. 5/6, S. $719-732$.

48 Vgl. Jürgen W. Falter, a.a.O. (Fn. 2), S. 62 f.

49 Vgl. Hans-Ulrich Wehler, a.a.O. (Fn. 7), S. 72. 